\title{
Evaluación de la satisfacción del paciente sometido a colonoscopia de escrutinio y la calidad del procedimiento
}

\author{
Evaluation of the satisfaction of the patient undergoing screening colonoscopy and \\ quality of the procedure
}

\author{
Óscar T. Teramoto-Matsubara ${ }^{1,2 *}$, Juan M. Abdo-Francis ${ }^{1,3}$, Gualberto Mateos-Pérez ${ }^{1,4}$, Graciela Morales ${ }^{1}$ \\ y Sergio Sobrino-Cossín $0^{1,4}$ \\ ${ }^{1}$ Clínica Gástrica, Centro Avanzado en Endoscopia y Estudios Funcionales; ${ }^{2}$ Práctica Médica de Gastroenterología, Centro Médico ABC; ${ }^{3}$ Servicio \\ de Endoscopia, Hospital Ángeles Acoxpa; ${ }^{4}$ Servicio de Endoscopia, Hospital Ángeles del Pedregal. Ciudad de México, México
}

\section{Resumen}

Antecedentes: La colonoscopia de escrutinio requiere parámetros de calificación para determinar su eficiencia en la detección de lesiones cancerosas o precancerosas. Sus limitantes son su rechazo, la dificultad durante su preparación o la falta de preparación. Objetivo: Evaluar la satisfacción del paciente y los criterios de calidad del estudio. Método: Se incluyeron 100 pacientes enviados para colonoscopia de escrutinio asignados aleatoriamente a uno de dos grupos de preparación colónica: polietilenglicol (PEG) o picosulfato de sodio ( $P$ ), para evaluar la satisfacción y la limpieza colónica. Otra evaluación fue por medio de llamada de refuerzo al momento de la preparación. Resultados: Con $P$ se tuvieron mejores tolerabilidad y calificaciones de satisfacción, en comparación con PEG. No hubo diferencia para la calidad de la limpieza, la tasa de detección de pólipos adenomatosos ni la intubación cecal. La llamada telefónica de reforzamiento incrementó la satisfacción, la limpieza colónica y la tasa de detección de adenomas. Discusión: Una relación estrecha con el paciente durante su proceso de limpieza y explicación del procedimiento se ha visto como uno de los factores más importantes para lograr una colonoscopia de alta calidad. Conclusión: El tipo de preparación colónica y la intervención durante la preparación se relacionaron con una mejor calidad del estudio y una mayor satisfacción del paciente.

Palabras clave: Calidad en colonoscopia. Cáncer colorrectal. Colonoscopia de escrutinio.

\begin{abstract}
Background: Screening colonoscopy requires quality parameters to determine its efficacy to detect cancerous or precancerous lesions. Limitations of the procedure are the patient's rejection, difficulty for its preparation or lack of preparation. Objective: To assess whether patient satisfaction correlates with the quality of the procedure. Method: 100 consecutive patients for a screening colonoscopy were included, they were randomized to use a preparation with polyethylene glycol (PEG) or sodium picosulfate $(P)$. In addition, a second parameter evaluated was related to a follow-up phone call performed to half of the patients prior to the study, during the preparation time. Results: $P$ was better tolerated and qualified, however, there was no difference with the quality of preparation, adenomatous polyps detection rate, cecum insertion rate and patient satisfaction. A difference was found in whom had a follow-up phone call. Discussion: A good colon preparation is a key factor to have a
\end{abstract}

\section{Correspondencia:}

*Óscar T. Teramoto-Matsubara

Avda. de Las Palmas, 735-1401

Col. Lomas de Chapultepec, Del. Miguel Hidalgo

Fecha de recepción: 20-10-2019

Cir Cir. 2020;88(4):441-447

C.P. 11700 , Ciudad de México, México

Fecha de aceptación: 25-03-2020

E-mail: teramotomd@prodigy.net.mx

DOI: $10.24875 / C I R U .20001666$

Contents available at PubMed

www.cirugiaycirujanos.com

0009-7411/@ 2020 Academia Mexicana de Cirugía. Publicado por Permanyer. Este es un artículo open access bajo la licencia CC BY-NC-ND (http://creativecommons.org/licenses/by-nc-nd/4.0/). 
quality colonoscopy, and it has been shown important that a good relationship for explain the doubts and follow the preparation. Conclusion: It was found that the type of preparation and patient follow-up during the study correlate with the quality of the study and patient satisfaction.

Key words: Quality colonoscopy. Colorectal cancer. Screening colonoscopy.

\section{Introducción}

La colonoscopia es un estudio diagnóstico y terapéutico cuya utilización se ha generalizado en nuestra sociedad. Los avances tecnológicos permiten que el procedimiento sea sencillo, seguro y efectivo para la detección oportuna y el tratamiento de las lesiones colorrectales.

Se ha reportado que los métodos de escrutinio reducen la mortalidad asociada al cáncer colorrectal. Sin embargo, existen diferencias entre las diferentes pruebas: mientras que la prueba de sangre oculta en heces no inmunoquímica se reporta que disminuye la mortalidad en un $33 \%$, la colonoscopia mejora esta probabilidad hasta en el 56\%1. Por ello, la colonoscopia se considera en la actualidad como el método estándar de referencia para el escrutinio².

Una colonoscopia de escrutinio es aquella en la cual no existe una indicación diagnóstica o de vigilancia. Probablemente, en estos casos, la preparación del colon es un factor determinante para lograr una colonoscopia exitosa, por lo que ha sido un aspecto de gran atención y estudio. La preparación inadecuada condiciona la probabilidad de pasar por alto y no diagnosticar lesiones importantes, y por lo mismo, de tener que repetir el examen hasta lograr una mejor limpieza. Una baja tasa de detección de adenomas se ha relacionado con un mayor riesgo de cáncer colorrectal de intervalo ${ }^{3,4}$.

Un intervalo de cáncer ha sido definido como el cáncer colorrectal diagnosticado durante el período de vigilancia (antes de la primera revisión) ${ }^{4}$.

La calificación para lograr el estándar de calidad en una colonoscopia de escrutinio incluye evaluar diversos aspectos técnicos, como el equipo de endoscopia o el uso de aditamentos adaptables ${ }^{3}$ que permiten mejorar la visión del colon, como son el endocuff y los endorings ${ }^{5}$, y por otro lado la satisfacción del paciente, para la cual se ha visto que la facilidad de la preparación es esencial. Estos aspectos se han vertido en diversas guías y recomendaciones ${ }^{6,7}$.

El objetivo de este estudio fue evaluar la calidad de los procedimientos de colonoscopia para escrutinio realizados en una clínica ambulatoria con respecto a dos tipos de preparación de limpieza colónica, el efecto de una intervención educativa (llamada telefónica de reforzamiento) y el grado de satisfacción del paciente.

\section{Método}

Se realizó un estudio prospectivo, comparativo y transversal en un servicio de endoscopia privado de la Ciudad de México. Se incluyeron, previo consentimiento escrito, 100 pacientes consecutivos de 50 a 75 años de edad, de ambos sexos, enviados a colonoscopia de escrutinio por primera vez. Se excluyeron aquellos con resección quirúrgica del colon, antecedente personal de cáncer colorrectal prevalente (activo o bajo tratamiento), datos de alarma (hemorragia digestiva baja, hematoquecia, acintamiento de heces, masa abdominal o pérdida de peso $>5 \mathrm{~kg}$ inexplicable en los últimos 3 meses), insuficiencia renal crónica, insuficiencia cardiaca congestiva u otra enfermedad que contraindicara una preparación del colon o que interfiriera con la comprensión de las órdenes o su motricidad, además de aquellos con alergias conocidas a alguno de los fármacos empleados antes, durante y tras el procedimiento.

El consumo de fármacos antiplaquetarios, como la aspirina o el clopidogrel, fue suspendido 7 días antes del procedimiento; aquellos pacientes en quienes no fue posible suspenderlo o que tomaban anticoagulantes no fueron incluidos.

A todos los sujetos se les dio una explicación detallada sobre las guías de calidad en colonoscopia y se les tomaron datos clínico-demográficos. En la evaluación inicial se les dio información verbal individualizada y por escrito sobre la preparación de limpieza colónica8.

El día previo al estudio, a todos los participantes se les recordó la cita y se resolvieron telefónicamente todas las dudas sobre la preparación colónica. Todos acudieron acompañados por un pariente, y se les efectuó una encuesta de satisfacción inmediatamente antes de su egreso y una llamada telefónica para confirmar el estado del paciente a las 8 horas del procedimiento. 
Los pacientes fueron asignados aleatoriamente para recibir en la preparación colónica polietilenglicol (PEG) o picosulfato de sodio (P). En ambos casos, la preparación fue a la dosis convencional fraccionada.

El día previo, los pacientes estuvieron bajo una dieta de abundantes líquidos claros, y en ayuno el día del estudio. Aleatoriamente, a la mitad de los sujetos de cada grupo se les efectuó una llamada telefónica de reforzamiento (intervención educativa) para resolver dudas sobre la preparación y la tolerabilidad.

Se aplicó un cuestionario de Likert de cuatro puntos para valorar la satisfacción y la tolerabilidad, al paciente y al médico que realizó el procedimiento, el cual se ha descrito en un estudio realizado previamente ${ }^{9}$. En resumen, se aplicó un cuestionario(Fig. 1) al momento del ingreso del paciente a la unidad de endoscopia, que incluía preguntas sobre la tolerabilidad, si presentó síntomas y la capacidad de ingerir el laxante, así como su satisfacción o apreciación subjetiva de la calidad de la atención sobre la preparación y el procedimiento endoscópico realizado.

Se realizaron mediciones basales, durante y tras el procedimiento de las constantes vitales (presión arterial, frecuencia cardiaca, frecuencia respiratoria, temperatura y saturación de oxígeno). La colonoscopia fue realizada por uno de cuatro endoscopistas con más de 10 años de experiencia en colonoscopia. La sedación con propofol intravenoso fue proporcionada por un anestesiólogo de acuerdo con sus requerimientos, quien suministró el oxígeno por puntas nasales a $3 \mathrm{l} / \mathrm{min}$.

En todos los casos se insufló aire en la colonoscopia, se intentó alcanzar el ciego y el tiempo de retiro del colonoscopio se determinó en $>6$ minutos. En caso de encontrar un pólipo durante la exploración, se registraron su situación y tamaño, y se resecó con técnica de polipectomía con asa o con resección endoscópica de mucosa. La pieza fue enviada para su evaluación histopatológica.

El grado de limpieza se evaluó utilizando la guía sobre calidad en endoscopia de la Fuerza en Conjunto de la American Society of Gastrointestinal Endoscopy (ASGE) y la American Gastroenterological Association (AGA) ${ }^{4}$, y se calificó como 1 (excelente), 2 (buena), 3 (regular) o 4 (mala).

De las variables medidas, la variable principal de desenlace fue la calidad de la preparación colónica (escala de cuatro puntos de la ASGE y AGA). Las otras variables fueron la edad, el sexo, el tabaquismo, el etilismo, la percepción de salud (variable dicotómica: buena o mala) y la percepción de riesgo para cáncer colorrectal (variable dicotómica: temor o no temor).

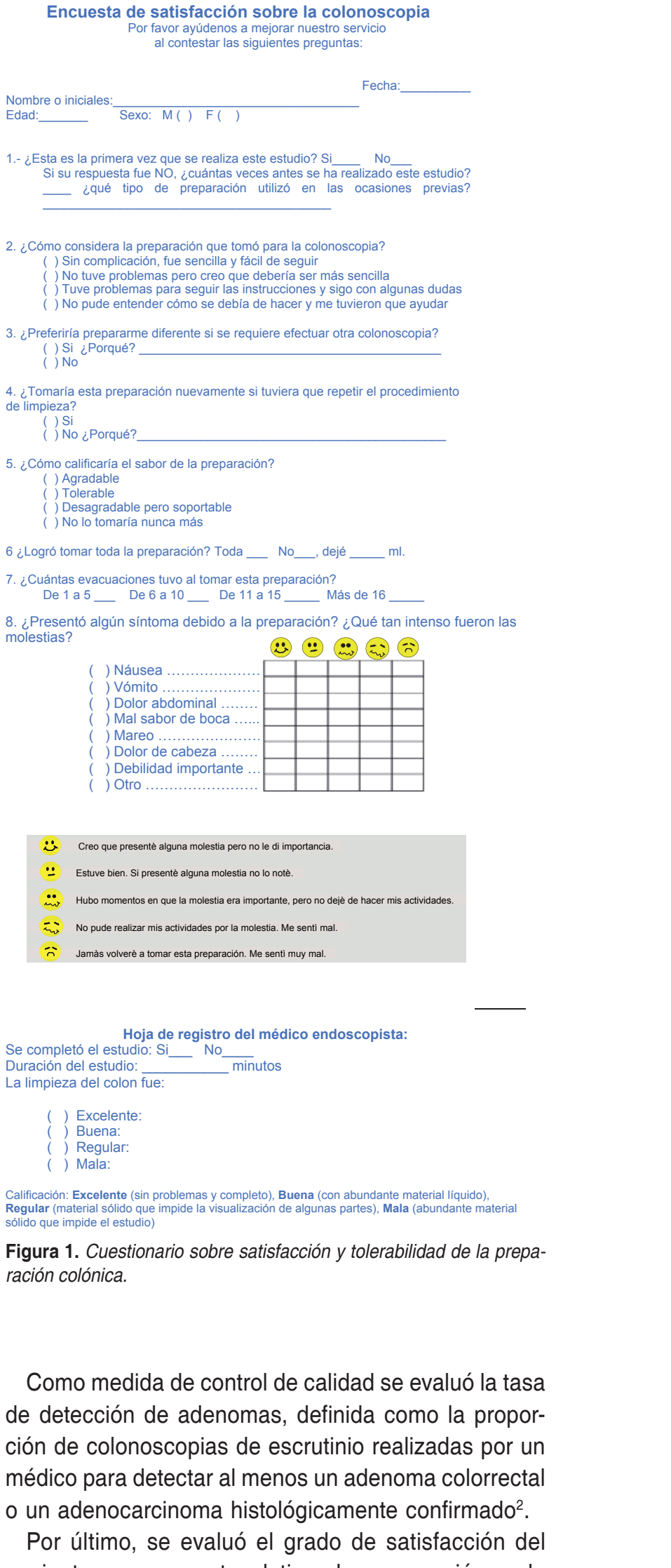
paciente con respecto al tipo de preparación y de seguimiento por el personal médico y paramédico. 


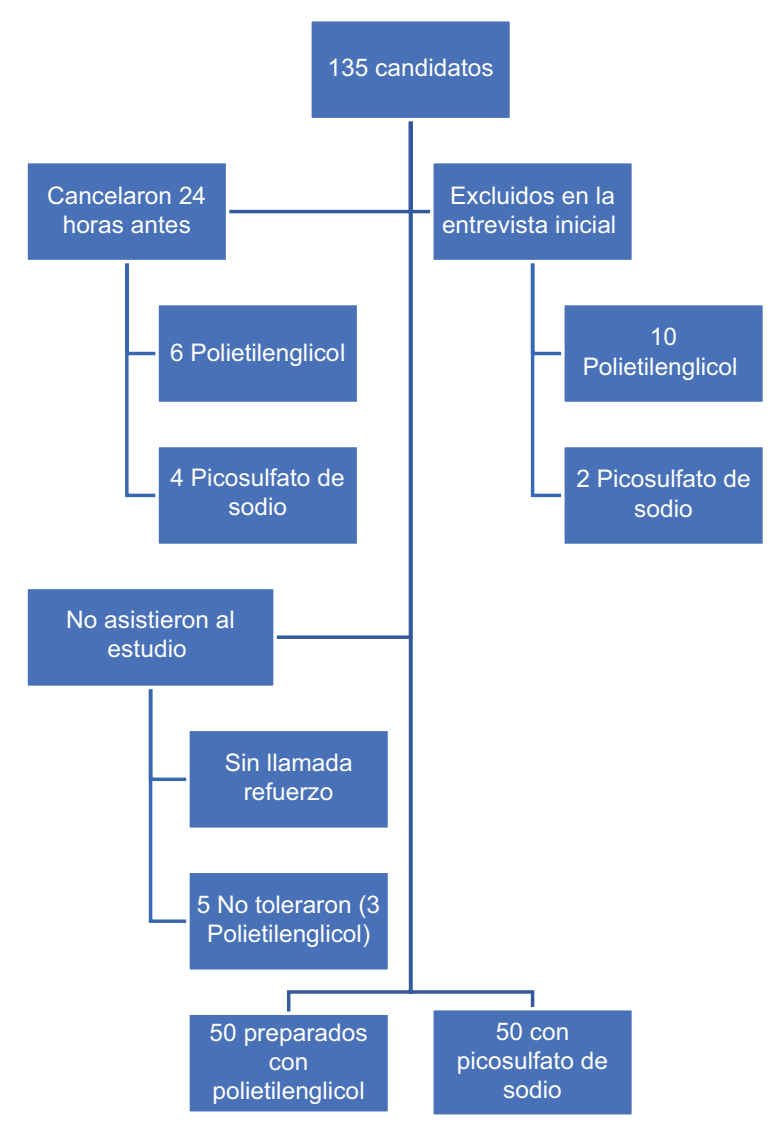

Figura 2. Diagrama de reclutamiento.

En el análisis estadístico, los datos clínico-demográficos se presentaron en forma descriptiva. Se realizaron las pruebas de ji al cuadrado y $t$ de Student para variables categóricas y cuantitativas, respectivamente. Se llevó a cabo un estudio binario de regresión logística para el grado de preparación entre pobre y regular frente a buena y excelente. Se utilizó un análisis binario de regresión logística para la tasa de detección de adenomas. Todo valor de $p<0.05$ en el análisis se consideró como estadísticamente significativo.

\section{Resultados}

Se entrevistó a 135 pacientes en un período de 7 meses. De ellos, 12 decidieron no realizarse el estudio en la encuesta inicial. Tras la aleatorización, 23 sujetos retiraron su consentimiento informado y no fueron incluidos en el estudio (Fig. 2).

No se realizó la colonoscopia por cancelación del procedimiento durante la llamada de confirmación el día previo en 10 casos (6 PEG y 4 P), en siete por intolerancia al fármaco (4 PEG y $3 \mathrm{P}$ ) y en seis por no asistir a la cita (en estos no hubo llamada de refuerzo).
Tabla 1. Características demográficas de los pacientes

\begin{tabular}{|c|c|c|c|c|}
\hline Variables & Total & Polietilenglicol & $\begin{array}{l}\text { Picosulfato } \\
\text { de sodio }\end{array}$ & $p$ \\
\hline Edad & $\begin{array}{c}57+18 \\
65 / 35\end{array}$ & $\begin{array}{c}58+13 \\
31 / 19\end{array}$ & $\begin{array}{c}56+19 \\
34 / 16\end{array}$ & $\begin{array}{l}\text { NS } \\
\text { NS }\end{array}$ \\
\hline Sexo (M/F) & 35 & 17 & 18 & NS \\
\hline $\begin{array}{l}\text { Diabetes } \\
\text { mellitus tipo } 2\end{array}$ & 28 & 16 & 12 & NS \\
\hline $\begin{array}{l}\text { Enfermedad } \\
\text { ácido-péptica }\end{array}$ & 15 & 8 & 7 & NS \\
\hline $\begin{array}{l}\text { Ingesta de } \\
\text { aspirina }\end{array}$ & 30 & 16 & 14 & NS \\
\hline
\end{tabular}

En el grupo de análisis final de 100 sujetos a quienes se realizó la colonoscopia, la edad promedio fue de 57 \pm 11 años y el $65 \%$ eran hombres. La distribución de los grupos y sus características se muestran en la tabla 1. No se encontraron diferencias entre los grupos.

El $4 \%$ eran bebedores de alcohol y el $30 \%$ eran fumadores ocasionales. La comorbilidad más común fue hipertensión arterial (35\%), diabetes mellitus tipo $2(28 \%)$ y enfermedad ácido-péptica (15\%). El uso de antiinflamatorios no esteroideos fue del $8 \%$, y de aspirina fue del $30 \%$. No hubo diferencias estadísticamente significativas entre los grupos.

En el $15 \%$ de la población femenina existió el antecedente de histerectomía por miomatosis o hemorragia vaginal (Tabla 1).

\section{Calificación de la preparación del colon con el tipo de laxante y satisfacción del paciente}

En el 95\% de los casos se logró la intubación del ciego y en el $94 \%$ se logró pasar la válvula ileocecal y revisar detenidamente el íleon terminal. Los tiempos de inserción (6.4 min con P vs. 7.18 min con PEG) y de retiro del colonoscopio (23.06 $\mathrm{min}$ con $\mathrm{P}$ vs. $23.10 \mathrm{~min}$ con PEG) fueron similares en ambos grupos.

La calidad de la limpieza colónica fue satisfactoria en el $95 \%$ e insatisfactoria en 5 casos (tres con $\mathrm{P}$ y dos con PEG) que no recibieron el día previo la intervención (llamada telefónica de refuerzo). Con la llamada de refuerzo se logró disminuir en 1 minuto el tiempo promedio de retiro del colonoscopio.

La calificación del endoscopista mostró una variación de excelente a buena preparación en el $88 \%$ en 
Tabla 2. Calidad de la preparación según el endoscopista

\begin{tabular}{|c|c|c|c|c|c|}
\hline & \multicolumn{2}{|c|}{ Picosulfato de sodio } & \multicolumn{2}{|c|}{ Polietilenglicol } & \multirow[t]{2}{*}{$p$} \\
\hline & Total (n) & Con refuerzo & Total & Con refuerzo & \\
\hline & 5 & 5 & 7 & 8 & 0.33 \\
\hline & 39 & 20 & 35 & 18 & \\
\hline & 5 & 0 & 6 & 0 & \\
\hline \multirow[t]{2}{*}{ Excelente } & 1 & 0 & 2 & 0 & \\
\hline & 50 & 25 & 50 & 25 & \\
\hline
\end{tabular}

Tabla 3. Satisfacción del paciente: facilidad de preparación y sabor

\begin{tabular}{|c|c|c|c|c|c|}
\hline \multirow[t]{2}{*}{ Calificación } & \multicolumn{2}{|c|}{ Picosulfato de sodio } & \multicolumn{2}{|c|}{ Polietilenglicol } & \multirow[t]{2}{*}{$p$} \\
\hline & Total & Con refuerzo & Total & Con refuerzo & \\
\hline & 19 & 12 & 13 & 6 & 0.18 \\
\hline & 27 & 17 & 24 & 15 & \\
\hline \multirow[t]{2}{*}{1} & 4 & 1 & 11 & 4 & \\
\hline & 2 & 1 & 0 & 0 & \\
\hline 2 & 50 & 31 & 50 & 25 & \\
\hline
\end{tabular}

ambos grupos; sin embargo, hubo diferencias en aquellos pacientes a quienes se hizo la llamada de reforzamiento en ambos grupos. Los casos con mala o regular preparación se presentaron en quienes no recibieron la llamada de reforzamiento, que llegó a ser del $32 \%$ en el grupo de PEG vs. $0 \%$, y del $24 \%$ en el grupo de $\mathrm{P}$ vs. $0 \%$ (Tabla 2).

La calidad de la preparación fue mejor calificada por los pacientes que tomaron $\mathrm{P}$, pero eso no mostró una diferencia significativa entre los grupos con respecto a los parámetros de limpieza, intubación al ciego o detección de pólipos ( $p=0.33$ ) (Tabla 3 ).

La intervención educativa permitió tener mayor contacto con el paciente y explicar la preparación en forma verbal y escrita. Por otra parte, la llamada telefónica de reforzamiento influyó significativamente en la calidad de colonoscopia. La tasa de detección de pólipos se incrementó del 28 al $47 \%$ con dicha intervención y fueron detectadas lesiones $<5 \mathrm{~mm}$ de diámetro (Tabla 4).

\section{Discusión}

Las actuales guías y consensos recomiendan que la colonoscopia es el método estándar de referencia para la detección de lesiones colorrectales en sujetos con riesgo promedio. Existe evidencia de que en sujetos asintomáticos la detección y la remoción de las lesiones precursoras (adenomas) mediante colonoscopia puede reducir la incidencia y reducir la mortalidad asociada a cáncer ${ }^{7,10,11}$.

De acuerdo con las guías europeas sobre el control de calidad en el escrutinio y diagnóstico del cáncer colorrectal $^{6}$, la calidad de la preparación del colon es un aspecto auditable en la colonoscopia y es un pilar para la evaluación del procedimiento. La preparación ideal debe cumplir varias características, entre las cuales las principales son que sea capaz de expulsar toda la materia fecal sin tener un efecto en la apariencia macroscópica del colon.

Existen reportes en la literatura sobre los efectos de una mala preparación del colon. En la actualidad, se considera el factor más importante para la eficacia y la calidad de la colonoscopia; una mala preparación incrementa el tiempo de su realización y, en especial, comporta una baja tasa de detección de adenomas ${ }^{7}$. Además, aumenta los costos del procedimiento y supone pérdida de tiempo, incremento de riesgos (aumento de cáncer colorrectal de intervalo) y necesidad de repetir el estudio ${ }^{8}$.

La calificación de los estándares de calidad al realizar una colonoscopia de escrutinio incluye la evaluación de aspectos técnicos, como es la experiencia del médico, el equipo de endoscopia, el tipo de luz y el uso de aditamentos para mejorar la visión de la mucosa del colon, como son las cubiertas llamadas endocuff y endorings, y por otro lado se encuentra la satisfacción del paciente, ya que la facilidad de la preparación es esencial para su aceptación y cooperación ${ }^{3,5}$.

Existen otros aspectos que se han sugerido como parámetros de calificación para la calidad, entre los que se encuentran las mediciones preprocedimiento (las indicaciones y el intervalo adecuado para el escrutinio), la tasa de intubación cecal, el tiempo de retiro del colonoscopio, la tasa de detección de adenomas, la adherencia a las guías, la satisfacción del paciente y la limpieza del colon 1,10,12.

La preparación del colon es una parte esencial, por lo que su evaluación y análisis han sido revisados por muchos autores y se enfatizan en las guías de recomendaciones. Independientemente de la opción de preparación, es importante recordar que la dosificación debe ser dividida ${ }^{8}$ para mejorar su efectividad, seguridad y tolerabilidad, y para reducir el número de eventos adversos, así como los costos. Por ello, el personal de salud debe estar familiarizado con el producto para poder explicarlo claramente a los pacientes ${ }^{13}$. 
Tabla 4. Pacientes con pólipos adenomatosos y calidad de la limpieza colónica

\begin{tabular}{lccccc}
\hline & Pacientes con adenomas & Con reforzamiento & Cáncer o pólipo $\mathbf{2} \mathbf{~ c m}$ & Pólipo de $\mathbf{5 - 1 5} \mathbf{~ m m ~}$ & Pólipos $<5 \mathbf{~ m m}$ \\
\hline Picosulfato de sodio & 22 & 13 & 2 & 10 & 10 \\
Polietilenglicol & 25 & 15 & 1 & 10 & 14 \\
\hline
\end{tabular}

Si la preparación no es bien tolerada, incluso si no es eficaz, no será ingerida y será mucho menos efectiva. El objetivo de dividir o fraccionar la dosis tiene efectos benéficos que influyen directamente sobre la limpieza de la mucosa y la tolerabilidad. Los tres criterios para una preparación intestinal adecuada están interrelacionados: eficacia, seguridad y tolerabilidad ${ }^{14}$.

La preparación inadecuada es predecible a un grado significativo (Tabla 3). Hay dos conjuntos de predictores, uno que abarca factores médicos y el otro que se relaciona con temas tales como el estatus socioeconómico, el nivel educativo, el tipo de seguro y la alfabetización en salud ${ }^{15}$.

Las explicaciones verbales, escritas, mediante un video o a través de una APP han sido parte de las diferentes estrategias ${ }^{15}$. En nuestro caso, utilizamos la explicación verbal y escrita, así como recordatorios mediante llamada telefónica de reforzamiento, y la variante utilizada fue realizar una llamada adicional al momento de la preparación. Esta sola estrategia ayudó a mejorar tanto la satisfacción del paciente como la calificación de la calidad del procedimiento.

Se ha reportado que el $20-40 \%$ de las preparaciones para limpieza colónica siguen siendo inadecuadas $^{16}$. La dosis dividida y la dosis en el mismo día son más eficaces que la toma la noche antes ${ }^{17,18}$. La dosis dividida es mejor tolerada que la dosificación de la noche anterior ${ }^{18}$.

Para evaluar la seguridad, la efectividad y la tolerabilidad se utilizaron dos tipos de preparaciones: P y PEG. La cantidad de solución y el sabor del PEG son factores que causan cierta resistencia por parte de los candidatos, incluso en el momento de la entrevista; sin embargo, se lograron los objetivos de seguridad y efectividad. Algunos obstáculos pueden evitarse mediante una explicación adecuada e individualizada al paciente. Aun con la preparación de $P$, que es más sencilla y de mejor sabor, se presentaron obstáculos durante su implementación que disminuyeron parte de la calificación de la preparación.

\section{Conclusiones}

El seguimiento estrecho del paciente que requiere una colonoscopia de escrutinio, desde la explicación de la preparación hasta buscar una metodología para su seguimiento, es un aspecto importante para tener una colonoscopia de calidad. Se deben buscar nuevas alternativas para una preparación que sea más sencilla en su ejecución y de mejor sabor.

\section{Conflicto de intereses}

Los autores declaran que no existe conflicto de intereses.

\section{Responsabilidades éticas}

Protección de personas y animales. Los autores declaran que los procedimientos seguidos se conformaron a las normas éticas del comité de experimentación humana responsable y de acuerdo con la Asociación Médica Mundial y la Declaración de Helsinki.

Confidencialidad de los datos. Los autores declaran que han seguido los protocolos de su centro de trabajo sobre la publicación de datos de pacientes.

Derecho a la privacidad y consentimiento informado. Los autores han obtenido el consentimiento informado de los pacientes y/o sujetos referidos en el artículo. Este documento obra en poder del autor de correspondencia.

\section{Bibliografía}

1. Doubeni CA, Corley DA, Quinn VP, Jensen CD, Zauber AG, Goodmanet $\mathrm{M}$, et al. Effectiveness of screening colonoscopy in reducing the risk of death from right and left colon cancer: a large community-based study. Gut. 2018;67:291-8.

2. Wong MCS, Ching JYL, Chan VCW, Lam TYT, Luk AKC, Tanget RSY, et al. Determinants of bowel preparation quality and its association with adenoma detection: a prospective colonoscopy study. Med (United States). 2016;95:1-8.

3. Castaneda D, Popov VB, Verheyen E, Wander P, Gross SA. New technologies improve adenoma detection rate, adenoma miss rate, and polyp detection rate: a systematic review and meta-analysis. Gastrointest Endosc. 2018;88:209-22.e11.

4. Rex DK, Petrini JL, Baron TH, Chak A, Cohen J, Deal SE, et al. Quality indicators for colonoscopy. Am J Gastroenterol. 2006;101:873-85.

5. Rex DK, Repici A, Gross SA, Hassan C, Ponugoti PL, Garcia JR, et al. High-definition colonoscopy versus Endocuff versus EndoRings versus full-spectrum endoscopy for adenoma detection at colonoscopy: a multicenter randomized trial. Gastrointest Endosc. 2018;88:335-44.e2.

6. Valori R, Rey J-F, Atkin WS, Bretthauer M, Senore C, Hoff G, et al. European guidelines for quality assurance in colorectal cancer screening and diagnosis. First Edition. Quality assurance in endoscopy in colorectal cancer screening and diagnosis. Endoscopy. 2012;44(Suppl 3):SE88-105. 
7. Bénard F, Barkun A, Martel M, von Rentelin D. Systematic review of colorectal cancer screening guidelines for average-risk adults: summarizing the current global recommendations Florence. World J Gastroenterol. 2013:24:124-38

8. Bartnik Meneses S, Kim HM, Schoenfeld P. Split-dose bowel preparation improves adequacy of bowel preparation and gastroenterologists' adherence to National Colorectal Cancer Screening and Surveillance Guidelines Stacy. World J Gastroenterol. 2018:24:716-24.

9. Teramoto-Matsubara O, Vargas JA, Comuzzie AG, López-Alvarenga JC. ¿Cuál es la preparación intestinal óptima para la colonoscopia en nuestro medio? Estudio comparativo entre la preparación con fosfato de sodio vs polietilenglicol. Endoscopia. 2007;19:339-45.

10. Min JK, Cha JM, Cho YK, Kim JH, Yoon SM, Im JP, et al. Revision of quality indicators for the Endoscopy Quality Improvement Program of the National Cancer Screening Program in Korea. 2018;51:239-52.

11. Restall G, Walker JR, Waldman C, Zawaly K, Michaud V, Moffat D. Perspectives of primary care providers and endoscopists about current practices, facilitators and barriers for preparation and follow-up of colonoscopy procedures: a qualitative study. BMC Health Serv Res. 2018;18:782-92.

12. Andrealli A, Paggi S, Amato A, Rondonotti E, Imperiali G, Lenoci N, et al. Educational strategies for colonoscopy bowel prep overcome barriers against split-dosing: a randomized controlled trial. United European Gastroenterol J. 2018:6:283-89.

13. Read AJ, Weissman A, Schoenfeld PS, Saini S, Menees SB, Saini SD. Who is responsible for what happens before, during, and after colonoscopy? Results of a National Survey of Primary Care Physicians. J Clin Gastroenterol. 2017;21:21.

14. Calderwood AH, Mahoney EM, Jacobson BC. A plan-do-study-act approach to improving bowel preparation quality. Am J Med Qual. 2017:32:194-200.

15. Gandhi K, Tofani C, Sokach C, Patel D, Kastenberg D, Daskalakis C. Patient characteristics associated with quality of colonoscopy preparation: a systematic review and meta-analysis. Clin Gastroenterol Hepatol. 2018:16:357-69.e10.

16. Radaelli F, Paggi S, Repici A, Gullotti G, Cesaro P, Rotondano G, et al. Barriers against split-dose bowel preparation for colonoscopy. Gut. 2017;66:1428-33.

17. Martin D, Walayat S, Ahmad Z, Dhillon S, Asche CV, Puli S, et al. Impact of bowel preparation type on the quality of colonoscopy: a multicenter community-based study. J Community Hosp Intern Med Perspect. 2016;6:31074

18. Gimeno-García AZ, Baute JL, Hernández G, Gonzalez-Pérez CD, Nicolás-Pérez D, Alarcon-Fernández $\mathrm{O}$, et al. Risk factors for inadequate bowel preparation: a validated predictive score. Endoscopy. 2017;49:536-43. 